\title{
In vitro evaluation of pan-PI3-kinase inhibitor SF1126 \\ in trastuzumab-sensitive and trastuzumab-resistant HER2-over-expressing breast cancer cells
}

\author{
Tuba Ozbay · Donald L. Durden · Tongrui Liu • \\ Ruth M. O'Regan · Rita Nahta
}

Received: 9 January 2009 / Accepted: 8 July 2009 / Published online: 28 July 2009

(C) The Author(s) 2009. This article is published with open access at Springerlink.com

\begin{abstract}
Purpose The purpose of the current study is to determine the in vitro cytotoxic effects of the novel pan-PI3-kinase inhibitor SF1126 in HER2-over-expressing breast cancer cells.

Methods Cell proliferation and cytotoxicity were examined by MTS colorimetric assay, FACS analysis, colony formation assay, and immunoblotting. Phosphoinositol-3-
\end{abstract}

T. Ozbay $\cdot$ R. Nahta $(\bowtie)$

Department of Pharmacology,

Emory University School of Medicine,

Suite 5001, 1510 Clifton Rd., Atlanta, GA 30322, USA

e-mail: rnahta@emory.edu

\section{L. Durden}

Department of Pediatrics,

Emory University School of Medicine,

Atlanta, GA 30322, USA

T. Liu $\cdot$ R. M. O’Regan $\cdot$ R. Nahta

Department of Hematology and Medical Oncology,

Emory University School of Medicine,

Atlanta, GA 30322, USA

T. Ozbay · D. L. Durden · T. Liu · R. M. O’Regan · R. Nahta

Winship Cancer Institute,

Emory University School of Medicine,

Atlanta, GA 30322, USA

\section{R. Nahta}

Molecular \& Systems Pharmacology Program,

Graduate Division of Biological and Biomedical Sciences,

Emory University School of Medicine, Atlanta, GA 30322, USA

D. L. Durden

Section of Pediatric Hematology/Oncology,

Aflac Cancer Center and Blood Disorders Services and Children's Healthcare of Atlanta, Emory University School of Medicine,

Atlanta, GA 30322, USA kinase signaling was assessed by immunoblotting for phosphorylated Akt. Combination effects of trastuzumab and SF1126 were examined in resistant cells by MTS and soft agar assay.

Results SF1126 inhibited proliferation, and induced G1 arrest and apoptosis of SKBR3 and BT474 parental and trastuzumab-resistant HER2-over-expressing cells. Colony formation was inhibited by SF1126, caspase 3 and PARP proteins were cleaved, and survivin was down-regulated. Inhibition of PI3-kinase was confirmed by reduced phosphorylation of Akt. Finally, the combination of SF1126 and trastuzumab synergistically inhibited proliferation of resistant cells, with SF1126-treated cells showing reduced anchorage-independent growth.

Conclusions These results provide evidence that a clinically relevant pan-PI-3 kinase inhibitor can reverse trastuzumab resistance in breast cancer cells, and support further study of PI3-kinase inhibitor SF1126 in HER2over-expressing breast cancer cells, including those that have progressed on trastuzumab.

Keywords Targeted therapy $\cdot$ Herceptin .

Drug resistance $\cdot$ PI3-kinase

\section{Introduction}

The human epidermal growth factor receptor 2 (her2) gene is amplified and/or over-expressed in approximately 20 $30 \%$ of invasive breast carcinomas, and is associated with increased metastatic potential and decreased overall patient survival [1]. Trastuzumab (Herceptin; Genentech, South San Francisco, CA) is a recombinant humanized monoclonal antibody directed against an extracellular domain of the HER2 tyrosine kinase receptor [2]. Clinical studies established 
that trastuzumab is active against HER2-over-expressing metastatic breast cancers [3-5], leading to its approval in 1998 by the US Food and Drug Administration. However, the objective response rates to trastuzumab monotherapy were low, ranging from 12 to $34 \%$ for a median duration of 9 months $[4,5]$. Even in combination with chemotherapy, response duration to trastuzumab is less than 1 year [6]. Thus, considerable effort has been made over the past decade to understand the mechanisms leading to trastuzumab resistance and to identify novel agents for patients with HER2-over-expressing metastatic breast cancer.

The anti-neoplastic effects of trastuzumab appear to require binding of the therapeutic antibody to HER2 with subsequent inhibition of downstream signaling through the phosphoinositol-3-kinase (PI3K)/Akt pathway. Constitutive Akt activity has been shown to block trastuzumab-mediated growth arrest and apoptosis in HER2-over-expressing breast cancer cells [7]. In addition, increased PI3K/Akt signaling due to downregulation of the phosphatase and tensin homolog (PTEN) or due to oncogenic mutations in the catalytic subunit of the PI3 kinase has been associated with reduced response to trastuzumab in patients with HER2-over-expressing breast cancer [8, 9]. Based on these findings, there is clear rationale for testing PI3K inhibitors in trastuzumab-resistant breast cancers.

Previous pre-clinical studies have suggested that Akt inhibition is a potentially effective strategy for HER2-overexpressing breast cancers that have progressed on trastuzumab. Triciribine, a synthetic tricyclic nucleoside that selectively inhibits phosphorylation of all Akt isoforms but does not inhibit upstream Akt activators such as PI3K [10], was shown to increase trastuzumab-mediated growth inhibition and tumor regression in PTEN-deficient trastuzumab-resistant breast cancer cells [11]. Another study described PTEN-deficient trastuzumab-resistant BT474 cell lines, and showed in vitro re-sensitization and in vivo antitumor effects of combination Herceptin plus PI3K inhibitor LY294002 [8]. However, despite these and other encouraging preclinical results, LY294002 is not a clinically viable agent, and thus could not be translated into practice.

In this study, we examined a clinically relevant small molecule inhibitor of all isoforms of PI3K (pan-PI3K inhibitor) called SF1126 (Semafore Pharmaceuticals, Indianapolis, IN) in HER2-over-expressing breast cancer cells and trastuzumab-resistant derivatives of these cells created by chronic, long-term exposure to trastuzumab $[12,13]$. SF1126 is a chemically modified form of PI3K inhibitor LY294002, with an RGDS peptide-linked integrin-targeted $(\alpha \mathrm{v} \beta 3 / \alpha 5 \beta 1$ targeted) group conjugated to the small molecule PI3K inhibitor. This modification is critical for increasing the water solubility and half-life of LY294002, hence facilitating clinical testing of this inhibitor [14]. SF1126 is currently in phase I trials for solid tumors including recurrent breast cancer and multiple myeloma. We show here that SF1126 inhibited proliferation and induced apoptosis of trastuzumab-naïve and trastuzumab-resistant HER2-over-expressing cells. In addition, the combination of SF1126 and trastuzumab showed synergistic anti-proliferative activity in trastuzumab-resistant cells. These results suggest that SF1126 should be examined further as a potential therapeutic for HER2-over-expressing breast cancers, including those that have become trastuzumab-resistant.

\section{Materials and methods}

\section{Materials}

Trastuzumab (Genentech; South San Francisco, CA) was obtained from the Emory Winship Cancer Institute pharmacy and dissolved in sterile water at a stock concentration of $20 \mathrm{mg} / \mathrm{ml}$. SF1126 was provided by Semafore Pharmaceuticals (Indianapolis, IN) to Dr. Donald L. Durden (Emory University) at a stock concentration of $40 \mathrm{mM}$ in water, and was used in phenol red-free cell culture media as previously described [14].

\section{Cell culture}

SKBR3 and BT474 parental cells were purchased from ATCC (Manassas, VA). Trastuzumab-resistant cells derived from SKBR3 and BT474 were developed as previously described $[12,13]$. Briefly, SKBR3 or BT474 cells were maintained in $4 \mu \mathrm{g} / \mathrm{ml}$ trastuzumab for 3 months, at which point the resistant pools were developed by collecting all viable cells on one plate; clones were developed by propagating a single colony of viable cells on a plate. Resistance to trastuzumab was confirmed by trypan blue exclusion or MTS proliferation assays and previously published $[12,13]$. Resistant cells are maintained on $4 \mu \mathrm{g} / \mathrm{ml}$ trastuzumab. All cells are maintained in Dulbecco's modified Eagle's medium supplemented with $10 \%$ fetal calf serum (FCS) and $1 \%$ penicillin/streptomycin, and incubated at $37^{\circ} \mathrm{C}$ with $5 \% \mathrm{CO}_{2}$ in humidified incubator.

\section{Dose-response assays}

Cells were plated at 5,000 per well in 96-well format, and treated with twofold serial dilutions of SF1126 for 6 days, at which point the cell proliferation was measured using the colorimetric MTS assay (Promega; Madison, WI). Experiments were done in six replicates and performed at least twice. Proliferation is expressed as a percentage of untreated cells per individual line; error bars represent standard deviation between replicates. 
Immunoblotting

Cells were lysed in RIPA buffer (Cell Signaling; Danvers, MA), which includes $0.1 \%$ NP40, supplemented with protease and phosphatase inhibitor cocktails (Sigma; St. Louis, MO). Total protein extracts $(50 \mu \mathrm{g})$ were run on SDSPAGE and immunoblotted using the following antibodies at the indicated dilutions: from Cell Signaling, p-Thr308Akt (polyclonal C31E5E) (1:500), p-Ser473-Akt (monoclonal 587F11) (1:200), polyclonal antibodies against total Akt, p-Thr202/Tyr204 p42/p44 MAPK (ERK1/2), total p42/p44 MAPK (ERK1/2), each used at 1:1,000, caspase 3 polyclonal (1:200), and PARP polyclonal used at 1:200; from Santa Cruz Biotechnology (Santa Cruz, CA), survivin monoclonal D-8 used at 1:500; and from Sigma, $\beta$-actin monoclonal used at 1:20,000. Secondary antibodies were chosen according to the species of origin of the primary antibody. Protein bands were detected using the Odyssey Imaging System (Li-Cor Biosciences).

\section{Cell cycle analysis}

Cells were untreated or treated with $40-\mu \mathrm{M}$ SF1126 in duplicate for $48 \mathrm{~h}$, fixed overnight in $50 \%$ ethanol, and then resuspended in PBS containing propidium iodide $(50 \mu \mathrm{g} /$ mL) supplemented with RNase A $(1 \mu \mathrm{g} / \mathrm{mL})$. DNA content was measured using a FACScan flow cytometer (Becton Dickinson, Franklin Lakes, NJ).

Colony inhibition assays

Cells were plated at 100,000 cells per 6-well. After $24 \mathrm{~h}$, cells were untreated or treated with $20-\mu \mathrm{M}$ SF1126 for 48 h. Drug-containing media were then removed, cells were washed with PBS, and drug-free media were added to cultures. Cells were maintained for an additional 6 days, at which point colonies were stained with $1 \%$ methylene blue. Imaging was performed using the Odyssey System (Li-Cor Biosciences; Lincoln, NE). Experiments were performed in duplicate at least twice.

Drug combination analysis

SK-HRp2 and BT-HRp3 cells were plated at 5,000 per well in 96-well format, and treated with SF1126 at 3.25, 7.5, 15, or $30 \mu \mathrm{M}$; trastuzumab at $1,2,4$, or $8 \mu \mathrm{g} / \mathrm{mL}$; or a combination of $\mathrm{SF} 1126+$ trastuzumab at $3.25 \mu \mathrm{M}+1 \mu \mathrm{g} / \mathrm{mL}$, $7.5 \mu \mathrm{M}+2 \mu \mathrm{g} / \mathrm{mL}, \quad 15 \mu \mathrm{M}+4 \mu \mathrm{g} / \mathrm{mL}$, or $30 \mu \mathrm{M}+8 \mu \mathrm{g} /$ $\mathrm{mL}$. After 6 days, MTS reagent was added per manufacturer (Promega) instructions. All samples were done in six replicates. Cell proliferation is expressed as a percentage of untreated cells per individual line; error bars represent standard deviation between replicates. Statistical analysis of drug combination effects and combination index (CI) was obtained using the method of Chou and Talalay [15] in the commercial software package Calcusyn (Biosoft, Cambridge, United Kingdom). CI $>1$ indicates antagonism between drugs, $\mathrm{CI}=1$ indicates additive effects, $\mathrm{CI}<1$ indicates drug synergy.

Soft agar assays

Anchorage-independent growth was assayed by colony formation on soft agar. Briefly, equal volumes of agar (1.2\%) and phenol red-free complete medium were mixed to make $0.6 \%$ agar growth medium solution in 6-well tissue culture plates. Cells $(50,000$ cells/well) were suspended in phenol red-free media with or without treatment followed by mixing with equal volume of agar $(0.6 \%)$. Cell suspension-agar mix $(2 \mathrm{~mL})$ was then added to each well. Plates were incubated at $37^{\circ} \mathrm{C}$ with $5 \% \mathrm{CO}_{2}$ in humidified incubator for 2 weeks, and media with or without treatment were added every 3 days. Colonies were stained with $0.01 \%$ crystal violet in PBS for $45 \mathrm{~min}$ at room temperature and observed using Olympus IX50 inverted microscope with $5 \times$ objective. Colonies that are equal to or bigger than average size from four randomly selected non-overlapping fields were counted.

\section{Results}

SF1126 inhibits proliferation of HER2-over-expressing breast cancer cells

SKBR3 trastuzumab-resistant pool 2 (SK-HRp2) and clone 3 (SK-HRc3) cells were developed by long-term treatment of HER2-over-expressing SKBR3 (SK-parental) breast cancer cells with trastuzumab, as previously described [12]. SK-parental, SK-HRp2, and SK-HRc3 were treated with increasing concentrations of SF1126 ranging from 7.5 to $120 \mu \mathrm{M}$ for 6 days. Proliferation was determined by MTS assay, and is expressed as a percentage of untreated cells per cell line (Fig. 1a). Proliferation was inhibited by SF1126 in a dose-dependent manner, with SK-parental and SK-resistant cells demonstrating similar responses with $\mathrm{IC}_{50}$ values of approximately $30-60 \mu \mathrm{M}$.

In addition to SKBR3-derived resistant cells, we generated BT474 trastuzumab-resistant pool 2 (BT-HRp2) and pool 3 (BT-HRp3) cells using the same approach as used to generate the trastuzumab-resistant SKBR3 cells [13]. BTparental, BT-HRp2, and BT-HRp3 cells were treated with SF1126 ranging from 7.5 to $120 \mu \mathrm{M}$ for 6 days. Cell proliferation was determined by MTS assay, and is expressed as a percentage of untreated cells per line (Fig. 1b). Similar to SKBR3 parental and resistant cells, BT474 cells displayed 

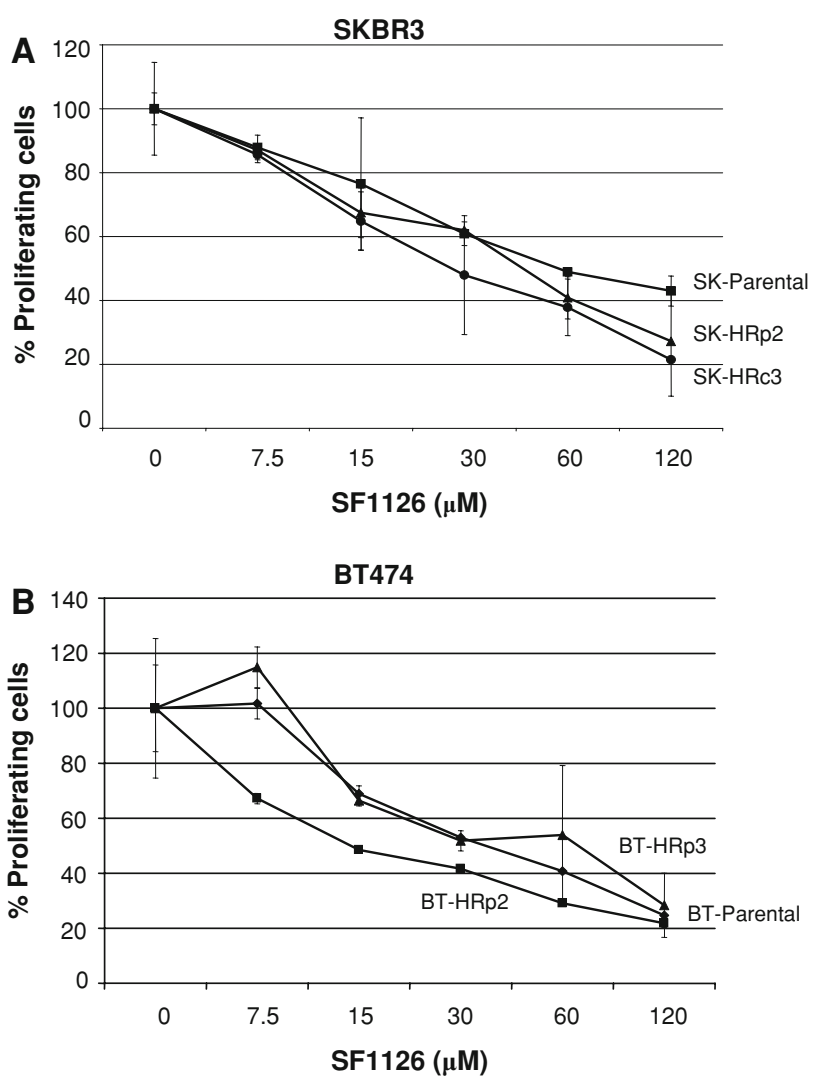

Fig. 1 SF1126 inhibits proliferation of parental and trastuzumabresistant HER2-over-expressing breast cancer cells. a Parental SKBR3 HER2-overexpressing breast cancer cells (SK-parental), SKBR3-derived trastuzumab-resistant pool 2 (SK-HRp2) and clone 3 (SK-HRc3), and b parental BT474 HER2-overexpressing breast cancer cells (BT-parental), BT474-derived trastuzumab-resistant pool 2 (BT-HRp2) and pool 3 (BT-HRp3) were treated with twofold serial dilutions of SF1126 ranging from 7.5 to $120 \mu \mathrm{M}$ for 6 days. Cell proliferation was measured using the MTS colorimetric assay, and is expressed as a percentage of untreated cells per line. Error bars represent standard deviation between six replicates. SF1126 inhibited the proliferation of parental and trastuzumab-resistant SKBR3 and BT474 HER2-overexpressing breast cancer cells

dose-dependent inhibition of proliferation. BT-parental and BT-HRp3 showed $\mathrm{IC}_{50}$ values of approximately $30 \mu \mathrm{M}$, while BT-HRp2 appeared to be slightly more sensitive with an $\mathrm{IC}_{50}$ of approximately $15 \mu \mathrm{M}-\mathrm{SF} 1126$.

SF1126 promotes G1 arrest and induces apoptosis of HER2-over-expressing cells

Cells were either untreated or treated with $40-\mu \mathrm{M}$ SF1126. After $48 \mathrm{~h}$, cells were fixed and stained with propidium iodide, and analysis of DNA content was performed by flow cytometry. Representative cell cycle profiles are shown for SK-parental and SK-HRp2 cells (Fig. 2a), and for BT-parental and BT-HRp3 cells (Fig. 2b). The percentage of SF1126-treated SK-parental and SK-HRp2 (Fig. 2c) and BT-parental and BT-HRp3 (Fig. 2d) cells in the proliferating fraction was reduced, and the percentage in G1 was increased, indicating G1 arrest. For all cell lines, SF1126 treatment resulted in an increase in the percentage of cells with sub-diploid DNA content (Fig. 3a). Parental cells showed approximately twofold increase in sub-diploid cells, while trastuzumab-resistant cells showed an eightfold increase in the percentage of sub-diploid cells, suggesting induction of apoptosis by SF1126.

SK-parental, SK-HRp2, BT-parental, and BT-HRp2 were treated with 0 or $20-\mu \mathrm{M}$ SF1126 for $48 \mathrm{~h}$. Cells were then maintained in drug-free media for an additional week, at which point colonies were stained with methylene blue (Fig. 3b). SF1126 treatment resulted in inhibition of colony growth, consistent with inhibition of proliferation and induction of cell death in both parental and resistant SKBR3 and BT474 cells.

To confirm that SF1126-mediated cell death was due to induction of apoptosis, SK-parental and SK-HRp2 cells (Fig. 3c) and BT-parental and BT-HRp2 cells (Fig. 3d) were treated with $0,10,20$, or $40-\mu \mathrm{M}$ SF1126 for $24 \mathrm{~h}$. Cleavage of poly (ADP-ribose) polymerase (PARP) and caspase 3, and expression of the anti-apoptotic molecule survivin were measured by immunoblotting. During apoptosis, caspase 3 is cleaved into 17 and $19-\mathrm{kDa}$ proteins, and PARP is cleaved by caspases to produce an $89-\mathrm{kDa}$ fragment from the full-length 116-kDa protein. SF1126 induced cleavage of PARP and caspase 3 in SKBR3 and BT474 parental and resistant cells consistent with induction of apoptosis. Consistent with the MTS proliferation assays, BT-parental and BT-HRp2 appeared slightly more sensitive than the SKBR3 parental and resistant cells, as cleavage of PARP and caspase 3 was more significant in the BT474 cells, especially when treated with the highest $40-\mu \mathrm{M}$ dose of SF1126. In addition, the anti-apoptotic molecule survivin was down-regulated in parental and resistant cells treated with SF1126. Together, these results indicate that SF1126 blocks proliferation with induction of G1 arrest and apoptosis in HER2-over-expressing breast cancer cells including those that are resistant to trastuzumab.

\section{SF1126 inhibits PI3K but not MAPK}

in trastuzumab-resistant cells

To assess the ability of SF1126 to inhibit its intended target (PI3K) in HER2-over-expressing breast cancer cells, immunoblotting was performed to detect phosphorylation of the PI3K substrate Akt. SK-parental, SK-HRp2, BTparental, and BT-HRp2 were treated with 10,20 , or $40-\mu \mathrm{M}$ SF1126 for $30 \mathrm{~min}$. Phosphorylation of Akt on serine 473 (S473) and threonine 308 (T308) is regulated by PI3K, 3-phosphoinositide-dependent protein kinase-1 (PDK-1), and mTOR. Thus, we assessed p-T308Akt and p-S473Akt as indicators of PI3K activity. Phosphorylation of S473Akt 


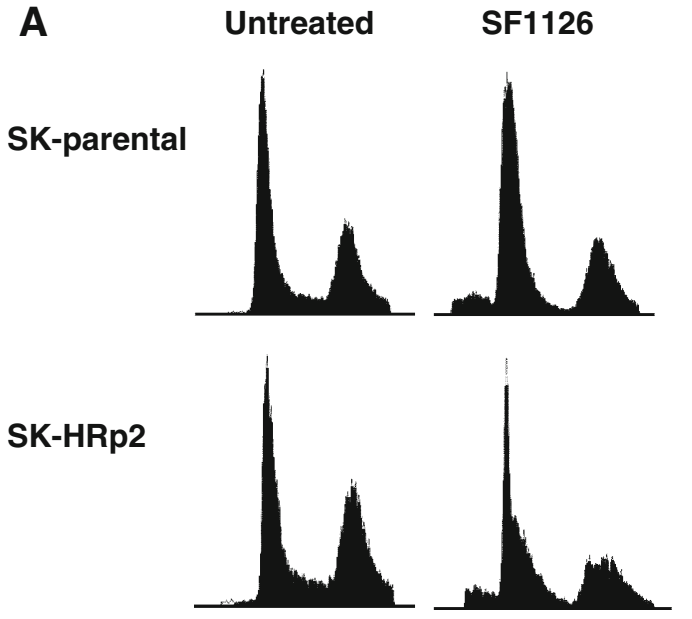

C
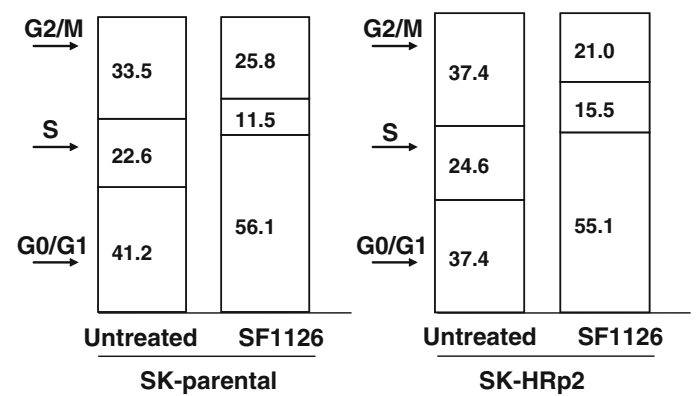

Fig. 2 SF1126 induces G1 arrest and inhibits proliferation. Cells were either untreated or treated with $40-\mu \mathrm{M} \mathrm{SF} 1126$ for $48 \mathrm{~h}$, at which point they were fixed and stained with propidium iodide. DNA content was then analyzed by flow cytometry. Representative cell cycle profiles are shown for a SK-parental and SK-HRp2, and b BT-parental and

was reduced with 40- $\mu$ M SF1126 in SKBR3 (Fig. 4a) and 10-20 $\mu$ M SF1126 in BT474 cells (Fig. 4c). Phosphorylation of T308 was inhibited with $10-20 \mu \mathrm{M}$ SF1126 in SKBR3 (Fig. 4b) and BT474 (Fig. 4d) cells. In contrast, ERK1/2 phosphorylation was not significantly affected, demonstrating relative specificity of SF1126 for Akt regulators including PI3 kinase, and possible inhibitory effects on PDK-1 and mTOR. BT474 cells appeared to be more sensitive to PI3K inhibition with reduced p-T308Akt and $\mathrm{p}-\mathrm{S} 473 \mathrm{Akt}$ levels achieved with $10-\mu \mathrm{M}$ SF1126. These results are consistent with proliferation assays, which showed stronger inhibition of proliferation by SF1126 in BT474 cells versus SKBR3 cells, with BT-HRp2 being more sensitive in that assay, and indicate that SF1126 inhibits its intended target (PI3-kinase) in HER2-overexpressing breast cancer cells.

Combination SF1126 and trastuzumab synergistically block proliferation of trastuzumab-resistant cells

SK-HRp2 (Fig. 5a) and BT-HRp3 (Fig. 5b) were treated with SF1126 at $3.25,7.5,15$, or $30 \mu \mathrm{M}$, trastuzumab at 1,2 ,

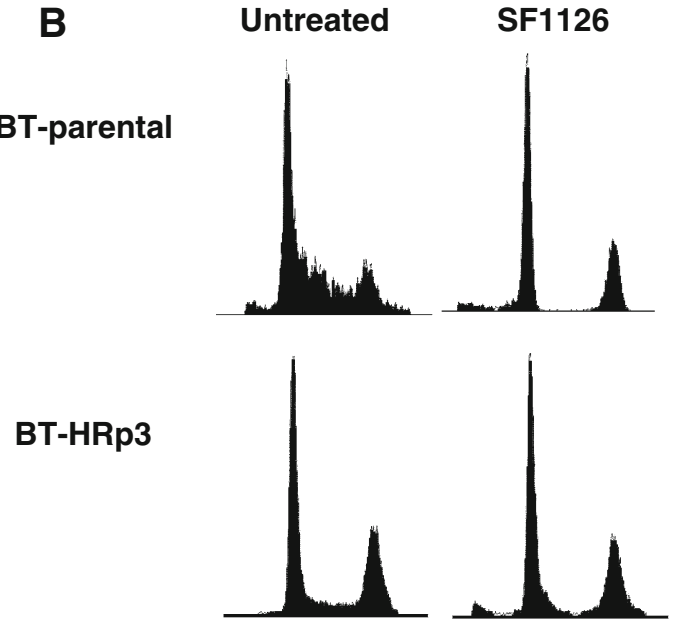

D
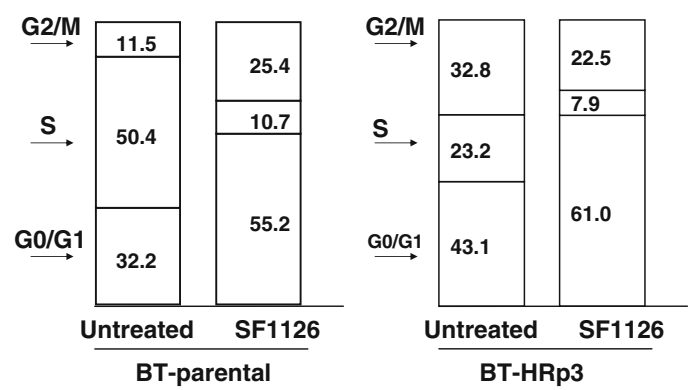

BT-HRp3 cells. The percentages of $\mathbf{c}$ SK-parental and SK-HRp2cells, and $\mathbf{d}$ BT-parental and BT-HRp3 cells in G0/G1, S, or G2/M are shown. SF1126 induced cell cycle arrest at the G1 phase with reduced $\mathrm{S}$ phase (proliferating fraction) in all cells

4 , or $8 \mu \mathrm{g} / \mathrm{mL}$, or a combination of the two drugs. Pharmacokinetic studies in vivo have confirmed that the concentrations of SF1126 used in these experiments are achieved in vivo in xenografted tumor tissues [14]. Proliferation was assessed by MTS assay after 6 days of treatment. Trastuzumab showed minimal to no inhibition of proliferation in resistant cells. SF1126 alone showed an $\mathrm{IC}_{50}$ of approximately $30 \mu \mathrm{M}$ in SK-HRp2 and $15 \mu \mathrm{M}$ in BT-HRp3. The combination of SF1126 and trastuzumab achieved an $\mathrm{IC}_{50}$ of less than $15 \mu \mathrm{M}$ in SK-HRp2 and approximately $3.25 \mu \mathrm{M}$ in BT-HRp3, suggesting that SF1126 increases trastuzumab activity in resistant cells. Statistical analysis of the combination dose-effect (Table 1) demonstrated CI values less than 1.0 in both SK-HRp2 and BT-HRp3, indicating drug synergy. The synergy was stronger in BT-HRp3, with $\mathrm{CI}$ values at low dose combinations ranging from 0.1 to 0.3 , versus SK-HRp2, which had CI values of approximately 0.7. The CI values at ED90 were likely higher because SF1126 had such high activity as a single agent, and reaching the effective dose at which $90 \%$ cells were killed (ED90) was likely already achieved by the higher doses of SF1126 used in these experiments. Overall, these 

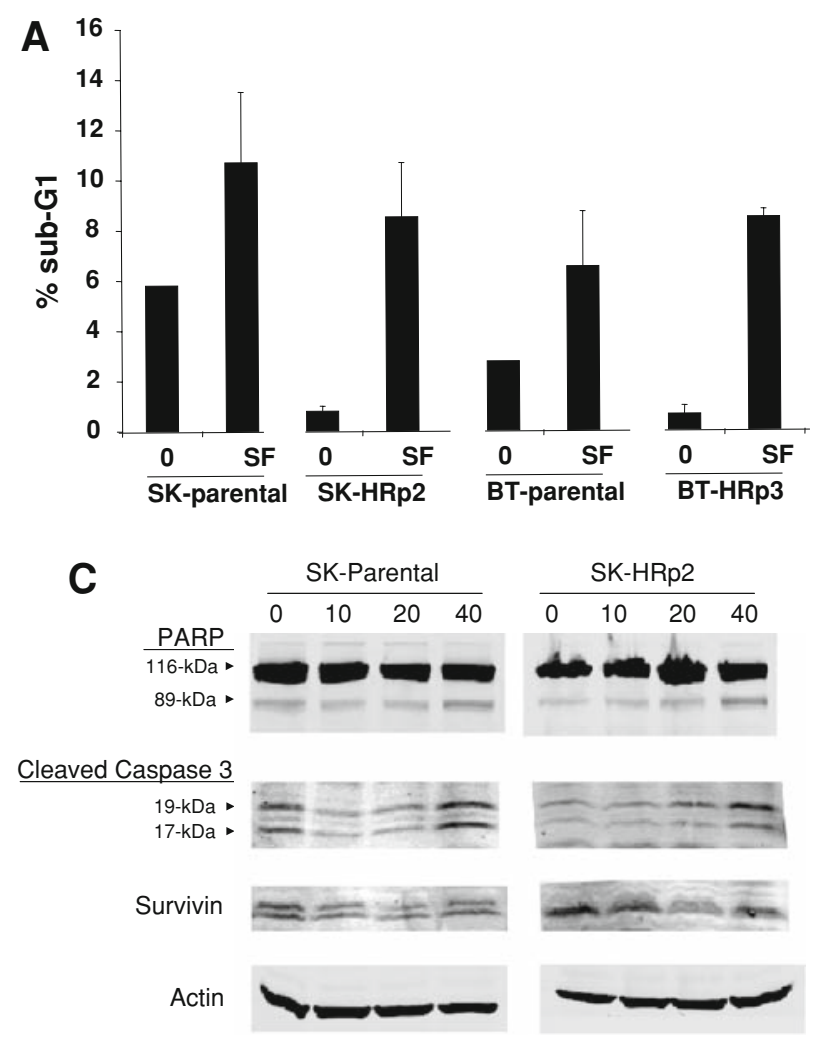

Fig. 3 SF1126 induces apoptosis of parental and trastuzumab-resistant cells. a SK-parental, SK-HRp2, BT-parental, and BT-HRp3 cells were either untreated or treated with $40-\mu \mathrm{M}$ SF1 126 for $48 \mathrm{~h}$, at which point they were fixed and stained with propidium iodide. DNA content was analyzed by flow cytometry. The percentages of cells with subdiploid DNA content are shown, with error bars representing standard deviation between duplicates. SF1126 increased the percentage of cells in sub-G1, consistent with induction of apoptosis. b Cells were untreated or treated with $20-\mu \mathrm{M} \mathrm{SF} 1126$ for $48 \mathrm{~h}$, at which point the drug-containing media were removed and replaced with drug-free media. Cells were maintained for an additional 6 days, then stained

results demonstrate that the combination treatment of trastuzumab plus SF1126 synergistically blocks proliferation of trastuzumab-resistant cells, and suggest that when used in combination, each drug may be administered at a lower dose versus when given as single agents.

In addition to proliferation assays, we examined the effect of combination treatment on anchorage-independent growth. Resistant cells were plated in soft agar with $15-\mu \mathrm{M}$ SF1126, $4 \mu \mathrm{g} / \mathrm{mL}$ trastuzumab, or a combination of both drugs for 2 weeks, at which point colonies were stained and counted in four random non-overlapping fields under a microscope. Trastuzumab showed minimal inhibition of colony growth, while SF1126 alone caused $50-90 \%$ growth inhibition (Fig. 5c). Combined trastuzumab plus SF1126 achieved slightly stronger inhibition of colony growth than SF1126 alone in all resistant cells. Thus, SF1126 appears to increase trastuzumab-mediated growth inhibition in HER2over-expressing breast cancer cells that have become resistant to trastuzumab.
B

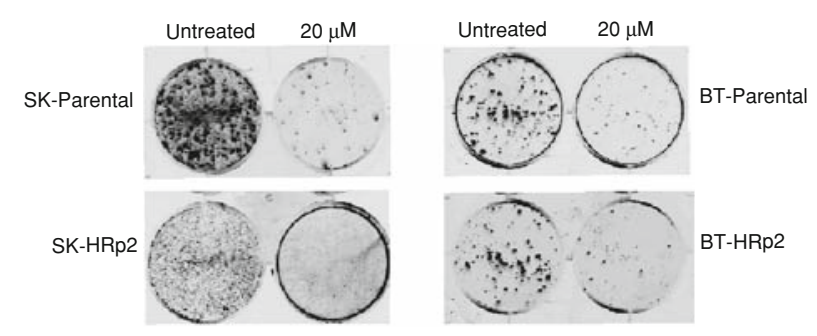

D

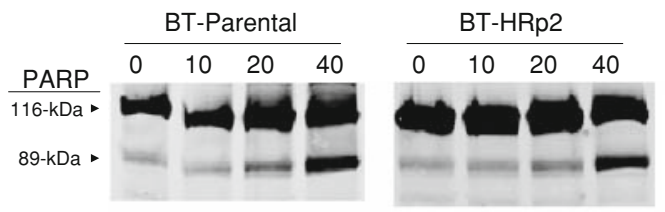

Cleaved Caspase 3
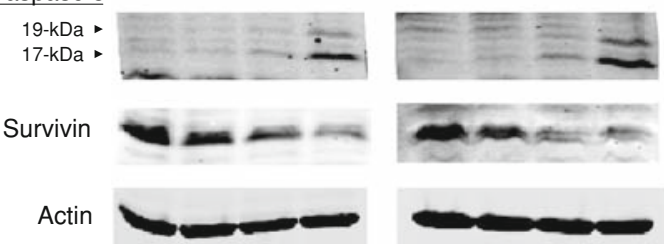

with methylene blue and photographed with the Odyssey Imaging System. Experiments were done in duplicate at least two times. Representative cultures are shown for each group. SF1126 inhibited colony survival. c SK-parental and SK-HRp2cells, and d BT-parental and BT-HRp2 cells were treated with $0,10,20$, or 40- $\mu \mathrm{M}$ SF1126 for $24 \mathrm{~h}$. Total protein lysates $(50 \mu \mathrm{g})$ were immunoblotted for PARP, cleaved caspase 3 , and survivin. Actin served as a loading control. SF1126 induced cleavage of PARP and caspase 3, and caused downregulation of survivin. These results indicate that SF1126 promotes growth arrest and apoptosis of HER2-over-expressing breast cancer cells

\section{Discussion}

In the current study, we examined the PI3K inhibitor SF1126 for activity against in vitro models of HER2-overexpressing breast cancer, including those that have developed trastuzumab resistance. SF1126 inhibited proliferation and colony formation, induced G1 arrest, and promoted apoptosis of HER2-overexpressing breast cancer cells, with relatively equal sensitivity between trastuzumab-naive and trastuzumab-resistant cells per line. SF1126 appeared to be specific for its intended target (PI3K), as Akt phosphorylation was reduced, while ERK1/2 phosphorylation was largely unaffected. Importantly, the combination of SF1126 and trastuzumab synergistically inhibited proliferation of trastuzumab-resistant cells, with combination indices less than 1.0. Anchorage-independent growth assays showed that SF1126 alone has strong inhibitory activity against trastuzumab-resistant cells, with addition of trastuzumab further increasing this inhibitory activity. Hence, SF1126 


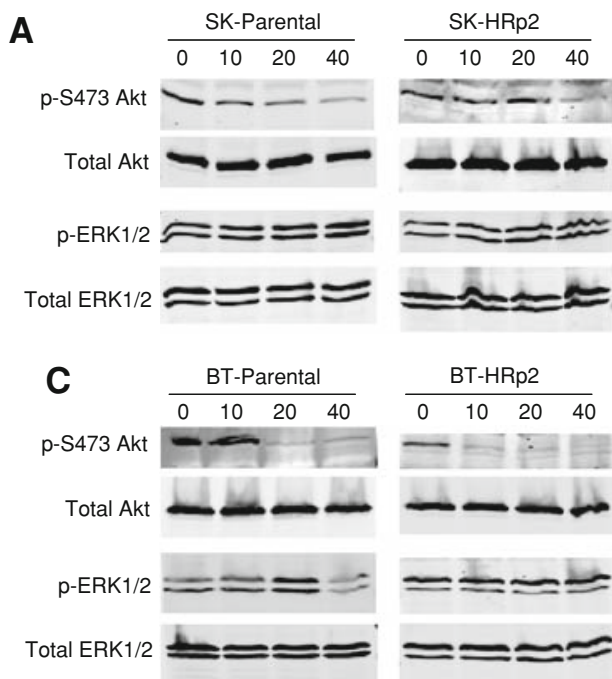

Fig. 4 SF1126 inhibits PI3K signaling. a, b SK-parental and SKHRp2, and c, d BT-parental, BT-HRp2 or BT-HRc3 cells were treated with $0,10,20$, or $40-\mu \mathrm{M}$ SF1126 for $30 \mathrm{~min}$. Total protein lysates $(50 \mu \mathrm{g})$ were immunoblotted for a, c phosphorylated serine 473 (p-S473) Akt, total Akt, phosphorylated Thr202/Tyr204 ERK1/2, and total ERK1/2, and for b, d phosphorylated threonine 308 (p-T308) Akt

appears to be an effective anti-cancer agent in HER2-overexpressing breast cancer cells, with activity retained in trastuzumab-resistant cells.

SF1126 is a chemically modified form of the commercially available PI3K inhibitor LY294002, with an RGDS peptide-linked integrin-targeted $(\alpha \mathrm{v} \beta 3 / \alpha 5 \beta 1$ targeted $)$ group conjugated to the small molecule PI3K inhibitor. The integrins $\alpha \mathrm{v} \beta 3$ and $\alpha 5 \beta 1$ are known to be expressed on the surface of neoplastic cells including SKBR3 cells [16], which are used in this study. As the increased efficacy of SF1126 versus LY294002 is believed to be due to the integrin-targeting RGDS peptide, cells that express these integrins should show increased response to SF1126 versus LY294002. If cells did not express the integrins, one may predict that cells will respond to SF1126 in a similar manner as to LY294002. Thus, we expect that the expression of integrins $\alpha v \beta 3 / \alpha 5 \beta 1$ on the cell surface increases response to SF1126. In addition, integrins are expressed on neovasculature [17], facilitating targeting of SF1126 to endothelial cells and allowing inhibition of angiogenesis [14]. Inhibition of angiogenesis, in addition to PI3K inhibition, may be an important mechanism of anti-tumor activity of SF1126 to be studied in animal models and clinical trials. Thus, the in vivo effects of SF1126 against trastuzumab-resistant breast cancer may be more pronounced than what we have observed in vitro.

SF1126 was the first pan-PI-3 kinase inhibitor to enter human clinical trials. It was shown in preclinical models to have single agent activity against breast cancer xenografts [14]. This agent is currently in phase I clinical trials in
B

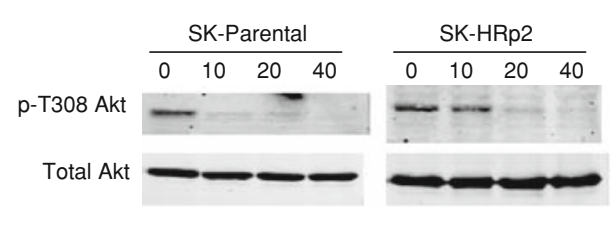

D

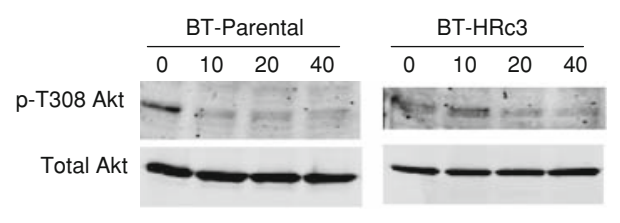

and total Akt. Actin served as a loading control. Parental and resistant SKBR3 cells showed reduced p-S473Akt with 40- $\mu$ M SF1126 and reduced p-T308Akt with lower doses of 10-20 $\mu \mathrm{M}$ SF1126. BT-parental and resistant cells showed reduced p-S473Akt and p-T308Akt at 10-20 $\mu \mathrm{M}$ SF1126. ERK1/2 phosphorylation was not significantly altered by SF1126

patients with breast, prostate, endometrial, and GIST tumors. Future studies will examine SF1126 and additional clinically relevant PI3K inhibitors using in vivo xenograft models of HER2-over-expressing trastuzumab-resistant breast cancer. These future studies will be necessary to establish strong rationale for clinical development of SF1126 and other PI3K inhibitors in the trastuzumab-resistant setting. In vivo studies will also allow us to determine dose-limiting toxicities of PI3K inhibition by SF1126 and doses required to achieve tumor response. Although doses of SF1126 required for in vitro activity were in the $\mu \mathrm{M}$ range, combination with trastuzumab allowed SF1126 doses to be reduced due to synergy between the two agents. Additionally, combination with chemotherapy would be expected to further decrease the dose of SF1126 required for in vivo effects. These studies are pending and will give critical information for future development of this novel PI3K inhibitor.

PI3K inhibition is an important molecular mechanism of the anti-cancer action of trastuzumab, as hyper-activation of PI3K signaling is associated with resistance to trastuzumab $[8,9]$. The specific molecular change inducing PI3K activity may vary among trastuzumab-resistant cancers, but has been reported to include PTEN loss [8], PI3K p110 subunit mutation [9], and increased activation of upstream growth factor receptors such as EGFR and IGF-IR [18-20]. Our results support the concept that PI3K inhibition is an important treatment approach for HER2-over-expressing breast cancers that have become resistant to trastuzumab. Previously published pre-clinical results showed 

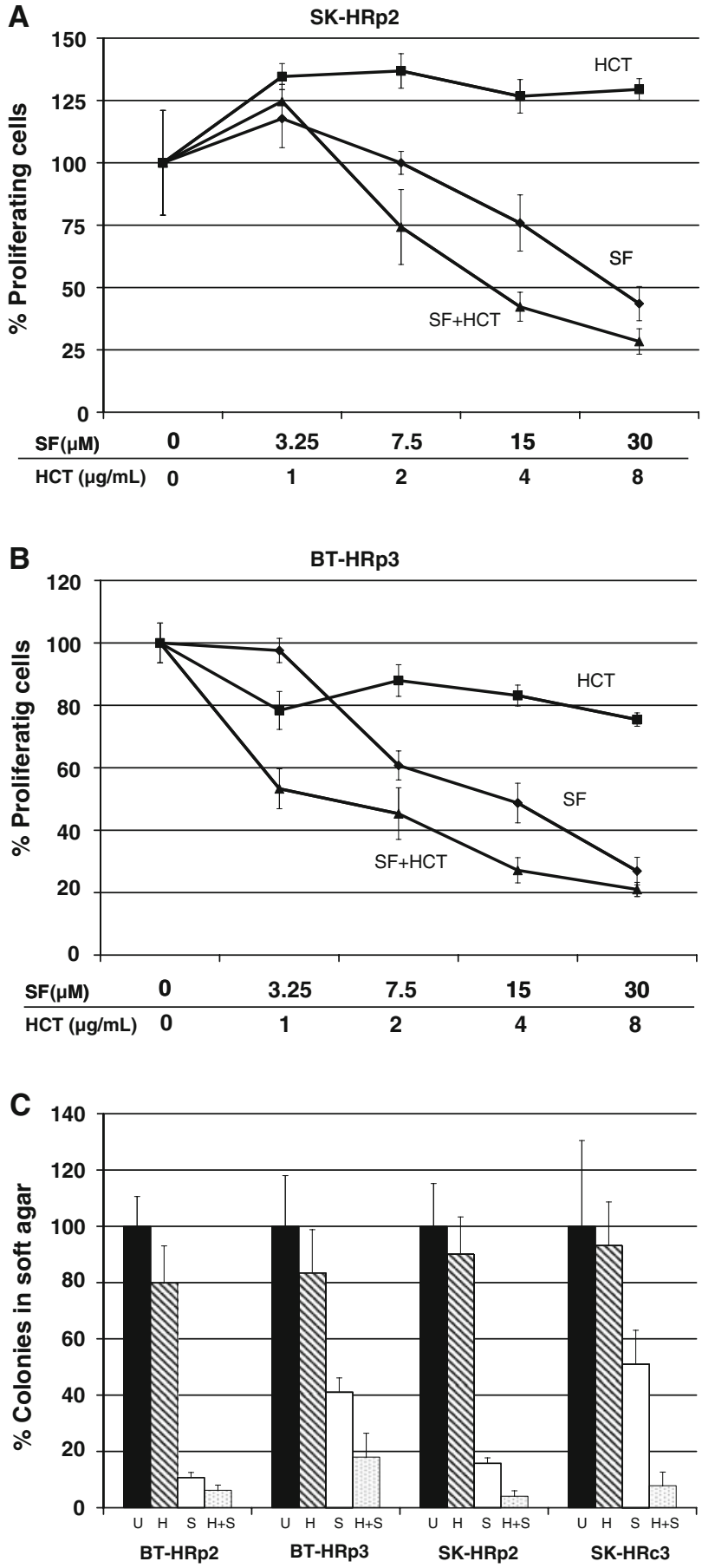

that combining trastuzumab with either the Akt inhibitor triciribine or the mTOR inhibitor Rad001 caused regression of trastuzumab-resistant tumors to a greater degree than either agent alone [11]. Our results showed that SF1126 and trastuzumab synergistically inhibit proliferation of trastuzumab-resistant cells. The implication of each of these findings is that trastuzumab resistance may not be due to permanent molecular changes, as PI3K inhibition restored trastuzumab activity at least to some degree. This is supported by results from a recent phase 1 clinical trial in which
Fig. 5 Combination SF1126 and trastuzumab synergistically inhibit proliferation of trastuzumab-resistant cells. a SK-HRp2 and b BTHRp3 cells were treated with $0,3.25,7.5,15$, or $30-\mu \mathrm{M}$ SF1126 (SF); $0,1,2,4$, or $8 \mu \mathrm{g} / \mathrm{mL}$ trastuzumab (HCT); or combination SF + HCT. After 6 days, MTS colorimetric assays were performed to measure cell proliferation. Values are expressed as a percentage of untreated cells per line. Error bars represent standard deviation between six replicates. The combination of trastuzumab with SF1126 inhibited proliferation of SK-HRp2 and BT-HRp3 more than SF1126 alone. (Results of statistical analysis are shown in Table 1.) c SK-HRp2, SK-HRc3, BTHRp2, and BT-HRp3 were plated in soft agar assays and untreated (U) or treated with trastuzumab $(\mathrm{H})$ at $4 \mu \mathrm{g} / \mathrm{mL}, \mathrm{SF} 1126(\mathrm{~S})$ at $15 \mu \mathrm{M}$, or the combination of drugs $(\mathrm{H}+\mathrm{S})$ at these doses. After 2 weeks of maintaining cells on drugs, colonies were stained with crystal violet and counted under a microscope in four random non-overlapping fields. The average number of colonies was calculated and is shown as a percentage of the average number of colonies in the untreated group per line. Error bars represent standard deviation of colony numbers between selected fields. SF1126 alone reduced colony growth by 50 $90 \%$, and combination with trastuzumab achieved stronger growth inhibition than SF1126 alone

Table 1 Combination index (CI) values for SF1126 plus trastuzumab in resistant cells

\begin{tabular}{llll}
\hline Cell name & ED50 & ED75 & ED90 \\
\hline SK-HRp2 & 0.61644 & 0.66352 & 0.73506 \\
BT-HRp3 & 0.28349 & 0.10304 & 4.52276
\end{tabular}

SK-HRp2 and BT-HRp3 cells were treated with $0,3.25,7.5,15$, or $30-\mu \mathrm{M}$ SF1126 (SF); 0, 1, 2, 4, or $8 \mu \mathrm{g} / \mathrm{mL}$ trastuzumab (HCT); or combination SF + HCT. After 6 days, MTS colorimetric assays were performed to measure cell proliferation. Statistical analysis of drug combination effects and CI was obtained using the method of Chou and Talalay [15] in the commercial software package Calcusyn (Biosoft, Cambridge, UK)

$\mathrm{CI}>1$ indicates antagonism between drugs, $\mathrm{CI}=1$ indicates additive effects, $\mathrm{CI}<1$ indicates drug synergy, ED 50 effective dose at which $50 \%$ cells are killed, ED75 effective dose at which $75 \%$ cells are killed, $E D 90$ effective dose at which $90 \%$ cells are killed

RAD001 appeared to reverse trastuzumab resistance in patients with metastatic HER2-positive cancers [21].

In addition to PI3K inhibition, several other combination treatment strategies have been proposed for trastuzumabresistant breast cancers. Lapatinib, a dual EGFR/HER2 kinase inhibitor, has been shown to be effective in pre-clinical models of trastuzumab resistance [22, 23]. The combination of lapatinib and trastuzumab has been demonstrated to have encouraging clinical activity in heavily pre-treated trastuzumab-resistant metastatic breast cancers [24]. Dual targeting of IGF-IR and HER2 is also a strategy of interest based on in vitro data suggesting possible up-regulation of IGF-IR expression or activation in trastuzumab resistance $[19,20]$. We previously reported that the combination of HER2 antibodies trastuzumab and 2C4 (pertuzumab) synergistically induce cell death of HER2-over-expressing BT474 breast cancer cells [25], and these promising results were recently confirmed in a phase 2 clinical trial [26]. As 
stated above, the combination of mTOR inhibition by RAD001 with paclitaxel and trastuzumab resulted in impressive activity in patients with heavily pre-treated trastuzumab-resistant metastatic breast cancer [21]. Finally, proteasome inhibition is a possible approach for restoring trastuzumab sensitivity based on pre-clinical results showing synergy between trastuzumab and the proteasome inhibitor bortezomib in HER2-overexpressing cells [27].

In conclusion, combining trastuzumab with additional targeted inhibitors may be an effective treatment strategy for trastuzumab-resistant breast cancers. Since PI3K is a central intercept node for a large number of signaling pathways in mammalian cells [17], many of which may be hyper-activated in trastuzumab resistance, combined HER2 and PI3K inhibition is an attractive therapeutic approach. Multiple clinically relevant PI3K or mTOR inhibitors are currently being developed and tested in combination with trastuzumab. Our study is the first to demonstrate that a pan-PI-3 kinase inhibitor that is in clinical development is effective in trastuzumab-resistant cells, and supports further analysis of PI3K inhibitor SF1126 as a single agent or in combination with trastuzumab in HER2-over-expressing breast cancers, including those that have progressed on trastuzumab-based therapy.

Acknowledgments We thank Dr. Laura M. Bender and Danica L. Rowe for their technical assistance with experiments. The authors gratefully acknowledge the following funding support: K01CA118174, National Cancer Institute (R Nahta); R01CA94233, National Cancer Institute (D Durden); Distinguished Cancer Scholar Award, Georgia Cancer Coalition (R Nahta, D Durden, R O'Regan); Wilbur and Hilda Glenn Foundation (R O'Regan)

Conflict of interest statement Dr. Durden would like to disclose a financial interest in Semafore pharmaceuticals. The terms of the arrangement have been reviewed and approved by Emory University in accordance with the conflict of interest policies.

Open Access This article is distributed under the terms of the Creative Commons Attribution Noncommercial License which permits any noncommercial use, distribution, and reproduction in any medium, provided the original author(s) and source are credited.

\section{References}

1. Slamon DJ, Clark GM, Wong SG et al (1987) Human breast cancer: correlation of relapse and survival with amplification of the HER-2/neu oncogene. Science 235:177-182

2. Carter P, Presta L, Gorman CM et al (1992) Humanization of an anti-p185HER2 antibody for human cancer treatment. Proc Natl Acad Sci USA 89:4285-4289

3. Baselga J, Tripathy D, Mendelsohn J et al (1996) Phase II study of weekly intravenous recombinant humanized anti-p185HER2 monoclonal antibody in patients with HER2/neu-overexpressing metastatic breast cancer. J Clin Oncol 14:737-744

4. Cobleigh MA, Vogel CL, Tripathy D et al (1999) Multinational study of the efficacy and safety of humanized anti-HER2 monoclonal antibody in women who have HER-2 overexpressing metastatic breast cancer that has progresssed after chemotherapy for metastatic disease. J Clin Oncol 17:2639-2648

5. Vogel CL, Cobleigh MA, Tripathy D et al (2002) Efficacy and safety of trastuzumab as a single agent in first-line treatment of HER2-overexpressing metastatic breast cancer. J Clin Oncol 20:719-726

6. Esteva FJ, Valero V, Booser D et al (2002) Phase II study of weekly docetaxel and trastuzumab for patients with HER-2-overexpressing metastatic breast cancer. J Clin Oncol 20:1800-1808

7. Yakes FM, Chinratanalab W, Ritter CA et al (2002) Herceptininduced inhibition of phosphatidylinositol-3 kinase and Akt is required for antibody-mediated effects on $\mathrm{p} 27$, cyclin D1, and antitumor action. Cancer Res 62:4132-4141

8. Nagata Y, Lan KH, Zhou X et al (2004) PTEN activation contributes to tumor inhibition by trastuzumab, and loss of PTEN predicts trastuzumab resistance in patients. Cancer Cell 6:117-127

9. Berns K, Horlings HM, Hennessy BT et al (2007) A functional genetic approach identifies the PI3K pathway as a major determinant of trastuzumab resistance in breast cancer. Cancer Cell 12:395-402

10. Yang L, Dan HC, Sun M et al (2004) Akt/protein kinase B signaling inhibitor-2, a selective small molecule inhibitor of Akt signaling with antitumor activity in cancer cells overexpressing Akt. Cancer Res 64:4394-4399

11. Lu CH, Wyszomierski SL, Tseng LM et al (2007) Preclinical testing of clinically applicable strategies for overcoming trastuzumab resistance caused by PTEN deficiency. Clin Cancer Res 13:58835888

12. Nahta R, Takahashi T, Ueno NT et al (2004) P27(kip1) down-regulation is associated with trastuzumab resistance in breast cancer cells. Cancer Res 64:3981-3986

13. Rowe DL, Ozbay T, Bender LM, Nahta R (2008) Nordihydroguaiaretic acid, a cytotoxic insulin-like growth factor-I receptor/HER2 inhibitor in trastuzumab-resistant breast cancer. Mol Cancer Ther 7:1900-1908

14. Garlich JR, De P, Dey N et al (2008) A vascular targeted pan phosphoinositide 3-kinase inhibitor prodrug, SF1126, with antitumor and antiangiogenic activity. Cancer Res 68:206-215

15. Chou TC, Talalay P (1984) Quantitative analysis of dose-effect relationships: the combined effects of multiple drugs or enzyme inhibitors. Adv Enzyme Regul 22:27-55

16. Burvenich I, Schoonooghe S, Vervoort L, Dumolyn C, Coene E, Vanwalleghem L, Van Huysse J, Praet M, Cuvelier C, Mertens N, De Vos F, Slegers G (2008) Monoclonal antibody 14C5 targets integrin alphavbeta5. Mol Cancer Ther 7:3771-3779

17. Castellino RC, Durden DL (2007) Mechanisms of disease: the PI3K-Akt-PTEN signaling node-an intercept point for the control of angiogenesis in brain tumors. Nat Clin Pract Neurol 3:682693

18. Ritter CA, Perez-Torres M, Rinehart C et al (2007) Human breast cancer cells selected for resistance to trastuzumab in vivo overexpress epidermal growth factor receptor and ErbB ligands and remain dependent on the ErbB receptor network. Clin Cancer Res 13:4909-4919

19. Lu Y, Zi X, Zhao Y et al (2001) Insulin-like growth factor-I receptor signaling and resistance to trastuzumab (Herceptin). J Natl Cancer Inst 93:1852-1857

20. Nahta R, Yuan LX, Zhang B et al (2005) Insulin-like growth factor-I receptor/human epidermal growth factor receptor 2 heterodimerization contributes to trastuzumab resistance of breast cancer cells. Cancer Res 65:11118-11128

21. André F, Campone M, Hurvitz SA et al (2008) Multicenter phase I clinical trial of daily and weekly RAD001 in combination with weekly paclitaxel and trastuzumab in patients with HER2-overexpressing metastatic breast cancer with prior resistance to trastuzumab. J Clin Oncol 26:May 20 suppl abstr 1003 
22. Konecny GE, Pegram MD, Venkatesan N et al (2006) Activity of the dual kinase inhibitor lapatinib (GW572016) against HER-2overexpressing and trastuzumab-treated breast cancer cells. Cancer Res 66:1630-1639

23. Nahta R, Yuan LX, Du Y et al (2007) Lapatinib induces apoptosis in trastuzumab-resistant breast cancer cells: effects on insulin-like growth factor I signaling. Mol Cancer Ther 6:667-674

24. O'Shaughnessy J, Blackwell KL, Burstein H et al (2008) A randomized study of lapatinib alone or in combination with trastuzumab in heavily pretreated HER $2+$ metastatic breast cancer progressing on trastuzumab therapy. J Clin Oncol 26:May 20 suppl abstr 1015
25. Nahta R, Hung MC, Esteva FJ (2004) The HER-2-targeting antibodies trastuzumab and pertuzumab synergistically inhibit the survival of breast cancer cells. Cancer Res 64:2343-2346

26. Gelmon KA, Fumoleau P, Verma S et al (2008) Results of a phase II trial of trastuzumab $(\mathrm{H})$ and pertuzumab $(\mathrm{P})$ in patients (pts) with HER2-positive metastatic breast cancer (MBC) who had progressed during trastuzumab therapy. J Clin Oncol 26:May 20 suppl; abstr 1026

27. Cardoso F, Durbecq V, Laes JF et al (2006) Bortezomib (PS-341, Velcade) increases the efficacy of trastuzumab (Herceptin) in HER-2-positive breast cancer cells in a synergistic manner. Mol Cancer Ther 5:3042-3051 